\title{
Influence du traitement des fourrages à l'ammoniac sur leur dégradation azotée dans le rumen
}

\section{B. Michalet-Doreau et C.V.M. Guedes *}

INRA, Centre de recherches de Clermont-Ferrand/Theix, station de recherches sur la nutrition des herbivores, unité de la valeur alimentaire, 63122 St-Genès, Champanelle, France

(reçu le 22 janvier 1989; accepté le 5 septembre 1989)

Résumé - La digestion de l'azote dans le rumen d'une paille, traitée avec de l'ammoniac anhydre $(5 \mathrm{~kg} / 100 \mathrm{~kg}$ ) ou non traitée, et d'un foin tardif traité également avec de l'ammoniac $(2 \mathrm{~kg} / 100 \mathrm{~kg})$ ou non traité, a été étudiée par la technique des sachets de nylon. Les teneurs en $\mathrm{N}$ ont augmenté après traitement respectivement de 11 et de $10 \mathrm{~g} / \mathrm{kg}$ de $\mathrm{MS}$, ce qui correspond à un taux de fixation de l'azote ammoniacal de $23 \%$ pour la paille et de $45 \%$ pour le foin.

Le traitement à l'ammoniac des fourrages a entraîné les modifications suivantes :

- la quantité d'azole rapidement dégradable (ou soluble) augmente dans des proportions importantes; elle passe respectivement de $4 \%$ pour la paille et $19 \%$ pour le foin à $62 \%$ pour les 2 fourrages traités;

- le pourcentage d'azote non dégradable n'est pas modifié par le traitement;

- la vitesse de dégradation de l'azote de la fraction potentiellement dégradable par les microorganismes du rumen diminue. Seulement $2 \%$ de l'azote initial est dégradé entre 0 et $1 \mathrm{~h}$ d'incubation dans le rumen pour les 2 fourrages traités.

L'azote fixé au cours du traitement est théoriquement entièrement dégradé dans le rumen $190 \%$ pour la paille et $100 \%$ pour le foin), mais l'azote disparaissant des sachets n'est peut-être pas entièrement utilisable par les microorganismes du rumen, ce qui expliquerait l'augmentation de l'azote excrété dans les fèces avec des fourrages traités.

paille — foin - traitement ammoniac — dégradabilité in sacco — valeur azotée

Summary - Influence of ammonia treatment of forages on nitrogen ruminal degradation. Ruminal nitrogen digestion of ammonia-treated or untreated straw $(5 \mathrm{~kg} / 100 \mathrm{~kg})$ and late-cut hay $(2 \mathrm{~kg} /$ $100 \mathrm{~kg}$ ) was studied using the in sacco technique. The post-treatment crude protein contents increased by 11 and $10 \mathrm{~g} / \mathrm{kg} \mathrm{DM}$, respectively, for straw and hay, corresponding to an ammonia nitrogen fixation rate of 23 and $45 \%$, respectively.

Ammonia treatment of forages led to the following modifications :

- the percentage of rapidly degradable (or soluble) nitrogen was greatly increased, from 4 and $19 \%$ for straw and hay to $62 \%$ for the 2 treated forages;

- the content of non-degradable nitrogen was not modified by the treatment;

- the nitrogen degradation rate of the potentially degradable fraction by rumen microbes decreased; for the 2 forages, only $2 \%$ of the initial nitrogen was broken down between 0 and $1 \mathrm{~h}$ of incubation in the rumen.

\footnotetext{
* Adresse actuelle : Université de Tras-os-Montes E Alto-Duro (UTAD), Vila Real (Portugal).
} 
Nitrogen bound during the treatment was theoretically fully degraded in the rumen 190 and $100 \%$ for straw and hay, respectively), but the nitrogen disappearing from the bags could perhaps not be completely used by the rumen microbes and this would account for the increase in faecal nitrogen excretion with the treated forages.

straw - hay - ammonia treatment - in sacco degradability - nitrogen value

\section{INTRODUCTION}

Le traitement des fourrages pauvres à l'ammoniac anhydre pour améliorer leur valeur alimentaire est largement utilisé actuellement pour les pailles de céréales (Sundstøl et al., 1978), et plus récemment pour les foins (Dulphy et al., 1984b). La plupart des travaux réalisés jusqu'ici ont mis en évidence l'effet positif du traitement à l'ammoniac sur la teneur en azote, les quantités ingérées et la valeur énergétique. Cependant, malgré l'enrichissement des fourrages en azote, l'augmentation de la valeur azotée est plus faible que prévue aussi bien pour la paille (Borhami et Johnson, 1981; Dulphy et al., 1984a) que pour le foin (Dulphy et al., 1984b; Benhamed et Dulphy, 1986). En effet, le traitement à l'ammoniac des foins et des pailles entraîne une augmentation anormale de l'azote excrété dans les fèces (Benhamed et Dulphy, 1985 et 1986; Ramihone, 1987). Celle-ci pourrait être due :

- soit à la fixation d'une partie de l'azote apporté par le traitement sur des parois indigestibles, ou non digérées dans le rumen, en raison de la baisse d'activité cellulolytique du rumen observée avec les rations à base de paille traitée (Chenost et Dulphy, 1987) ou de l'augmentation de la vitesse de transit ruminal (Steg et den Boer, 1982);

- soit au fait qu'une partie des protéines du fourrage est rendue indigestible par suite de l'échauffement dû au traitement (effet Maillard) (Solaiman et al., 1979);

- soit au fait qu'une partie de l'azote fixé lors du traitement n'est pas utilisable par les micro-organismes du rumen.

\section{MATÉRIEL ET MÉTHODES}

\section{Fourrages}

Nous avons utilisé une paille de blé et un foin de 1 er cycle de prairie permanente récolté tardivement et pressé à $75 \%$ de matière sèche. Dès leur récolte, les fourrages ont été traités à l'ammoniac anhydre selon la technique en meules bâchées hermétiques, à raison de $5 \mathrm{~kg}$ d'ammoniac par $100 \mathrm{~kg}$ de fourrage brut pour la paille et de $2 \mathrm{~kg}$ d'ammoniac par $100 \mathrm{~kg}$ pour le foin.

\begin{abstract}
Animaux
Trois vaches taries munies d'une canule du rumen recevant une ration à base de foin de dactyle $(70 \%)$ et de concentré ${ }^{*}(30 \%)$, à raison de $7 \mathrm{~kg}$ de MS/animal/jour, ont été utilisées pour les mesures de dégradation in sacco dans le rumen.
\end{abstract}

- Composition du concentré (en \% MS) : orge (38), pulpe de betterave (41), tourteau de soja (10), urée (2), minéraux + oligo-éléments (9). 


\section{Dispositif expérimental}

Les 4 fourrages (foin et paille, non traité et traité à l'ammoniac) ont été broyés à la grille de 0,8 $\mathrm{mm}$ et introduits dans des sachets en tissu de nylon de $46 \mu \mathrm{m}$ de taille de pores, à raison de $3 \mathrm{~g}$ de fourrage par sachet (Michalet-Doreau et al., 1987). Les dimensions externes et internes des sachets étaient respectivement de 7,5-15 $\mathrm{cm}$ et 6-11 cm, soit un rapport poids d'échantillon/surface de $20 \mathrm{mg} / \mathrm{cm}^{2}$.

Chaque série d'incubation comprenait 6 points de cinétique, soit 2-4-8-16-24 et $48 \mathrm{~h}$ d'incubation, et à chaque point de cinétique correspondaient 6 mesures ( 3 vaches $\times 2$ répétitions). Après incubation, les sachets ont été battus pendant $7 \mathrm{~min}$ dans un "stomacher", puis lavés à l'eau froide et séchés (Ould-Bah et al., 1988).

Le traitement au "stomacher" avait pour effet non seulement de débarasser les sachets des particules alimentaires ou microbiennes présentes sur le tissu, mais aussi de décrocher le maximum de bactéries fixées sur les particules alimentaires restant dans le sachet.

\section{Analyses}

Pour chaque fourrage, nous avons déterminé les teneurs en azote (méthode Kjeldahl), en parois (NDF) et en constituants ligno-cellulosiques (ADF) (Goering et Van Soest, 1970). La teneur en azote des résidus des sachets a également été déterminée par la méthode Kjeldahl, après regroupement des 2 sachets correspondant à un même point de cinétique et un même animal.

Par ailleurs, nous avons cherché à préciser la forme de l'azote des fourrages. Pour cela, nous avons dosé, sur les 4 fourrages, l'ammoniac libéré par extraction aqueuse à $+4^{\circ} \mathrm{C}$ pendant $18 \mathrm{~h}\left(\mathrm{~N}-\mathrm{NH}_{3}\right)$ (méthode de Conway, rapportée par Dulphy et Demarquilly, 1981), et nous avons déterminé le pourcentage d'azote solubilisé dans un tampon (azote soluble par la méthode Durand, rapporté par Vérité et Demarquilly, 1978), dans des détergents neutre ( $N$ NDS) ou acide (N-ADS).

\section{Calculs}

Malgré l'utilisation du "stomacher" pour diminuer la colonisation des sachets par les bactéries du rumen, une quantité de matière sèche bactérienne notablement réduite $(<4 \%$ de la matière sèche résiduelle) mais non négligeable, reste encore présente dans les sachets. Mais ce pourcentage de matière sèche bactérienne étant indépendant de la nature du fourrage, il est possible de calculer pour chaque point de cinétique, la quantité d'azote alimentaire résiduel à partir de la quantité d'azote résiduel après passage au stomacher et de ce pourcentage de matière sèche bactérienne (Ould-Bah et al., 1988).

Pour calculer la dégradabilité de l'azote alimentaire dans le rumen, les résultats de dégradation par aliment et par animal ont été ajustés à un modèle exponentiel décrit par Orskov et McDonald (1979), à savoir :

$$
D(t)=a+b(1-\exp (-c t))
$$

qui suppose 3 fractions azotées : une fraction immédiatement dégradable (a); une autre dont la dégradation se fait plus lentement $(b)$ avec une vitesse qui diminue exponentiellement

$(\exp (-c t))$; et une fraction non dégradable

$$
(100-a-b) \text {. }
$$

Pour comparer la dégradabilité de l'azote des fourrages dans le rumen, un taux de passage des particules alimentaires hors du rumen de 0,06 par heure a été retenu (Vérité et al., 1987) et la dégradabilité de l'azote $(D)$ a été calculée à l'aide de la formule suivante :

$$
D=a+b c /(c+0,06)
$$

\section{RÉSULTATS}

La composition chimique des fourrages avant et après traitement est donnée dans le Tableau I. Les teneurs en $\mathbf{N}$ de la paille et du foin ont augmenté après traitement, respectivement de 11 et de $10 \mathrm{~g} / \mathrm{kg}$ de MS, ce qui correspond à un taux de fixation de l'azote de $23 \%$ pour la paille et de $45 \%$ pour le foin. 
Tableau I. Composition chimique des fourrages avant et après traitement à l'ammoniac.

\begin{tabular}{lllll}
\hline & MS $(\%)$ & NT & $\begin{array}{c}\text { NDF } \\
(g / \mathrm{kg} \mathrm{MS})\end{array}$ & ADF \\
\hline $\begin{array}{llll}\text { Paille } \\
\text { non traitée }\end{array}$ & 92,6 & 6,6 & 82,6 & 58,6 \\
traitée & 92,1 & 17,8 & 73,7 & 55,3 \\
Foin & & & & 38,2 \\
non traité & 89,3 & 17,8 & 56,4 & 39,9 \\
traité & 88,3 & 27,7 & 58,3 & \\
\hline
\end{tabular}

Le traitement à l'ammoniac n'a pas modifié les teneurs en constituants pariétaux du foin, mais la teneur en parois de la paille a diminué de $89 \mathrm{~g} / \mathrm{kg}$ de MS, soit de $11 \%$. La diminution observée est principalement liée à celle des hémicelluloses, respectivement $56 \mathrm{~g} / \mathrm{kg}$ de MS pour les hémicelluloses contre $33 \mathrm{~g} / \mathrm{kg}$ de MS pour la fraction ligno-cellulosique.

Les différents constituants de la fraction azotée des fourrages sont donnés dans le Tableau II. Le traitement a entraîné une augmentation très importante de la fraction soluble dans un tampon, qui passe de 1,9 à $10,2 \mathrm{~g} / \mathrm{kg}$ de MS pour la paille et de 8,4 à 17,4 pour le foin. En proportion de l'azote fixé, on retrouve $91 \%$ de l'azote sous forme soluble pour le foin traité à l'ammoniac, dont $89 \%$ sont constitués d'azote ammoniacal. Pour la paille traitée, la fraction d'azote fixé qui est sous forme soluble, reste élevée $(75 \%)$, mais cependant plus faible que pour le foin et l'azote, encore sous forme d'ammoniac, ne représente plus que $55 \%$ de cette fraction, le reste se trouvant sous une forme indéterminée.
Quant à la fraction azotée qui n'est pas solubilisée par un détergent neutre $(\mathrm{N}$ NDF), elle n'est pas modifiée par le traitement à l'ammoniac pour le foin $(7,1 \mathrm{~g} / \mathrm{kg}$ de MS contre $7,9 \mathrm{~g} / \mathrm{kg}$ de MS), mais elle augmente pour la paille traitée; elle passe de 1,9 à $4,4 \mathrm{~g} / \mathrm{kg}$ de MS après traitement, et cette augmentation est d'ailleurs d'autant plus importante que parallèlement la teneur en NDF des pailles traitées a diminué. $23 \%$ de l'azote fixé au cours du traitement de la paille se retrouve sous forme d'azote lié à la fraction NDF, et seulement $7 \%$ est lié à la fraction ADF. Enfin, quel que soit le fourrage considéré, foin ou paille, traité à l'ammoniac ou non traité, la fraction d'azote qui n'est pas solubilisée par la pepsine est toujours plus importante que la fraction d'azote liée à l'ADF.

La quantité d'azote immédiatement dégradable dans les sachets qui est souvent assimilée à la quantité d'azote solubilisée dans le rumen, est presque nulle pour la paille, et relativement faible pour le foin ( $3,4 \mathrm{~g} / \mathrm{kg}$ MS) (Tableau III); par opposition, la quantité d'azote potentiellement dégradable par les micro-organismes du rumen 
Influence du traitement des fourrages à l'ammoniac

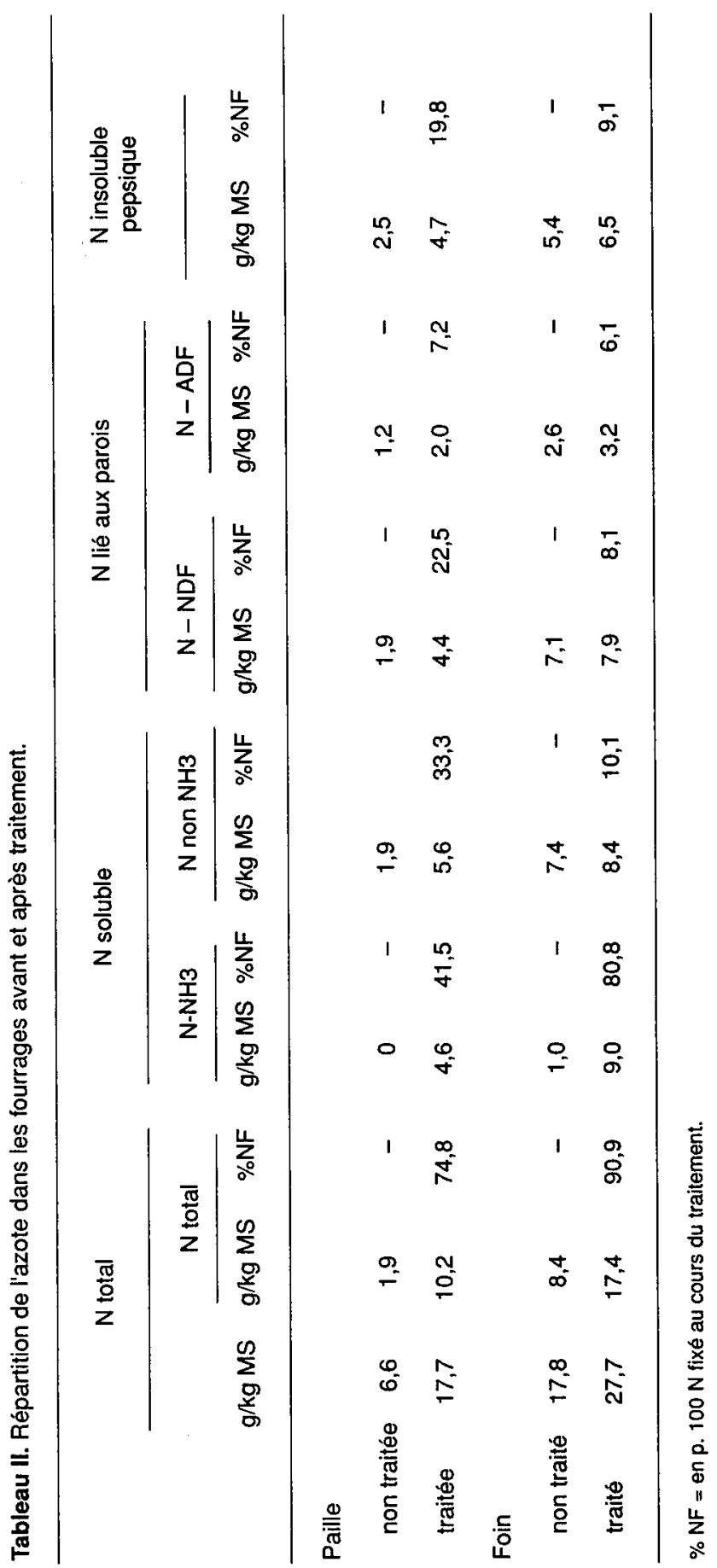


est importante et représente à elle seule $75 \%$ de l'azote total pour les 2 fourrages (Fig. 1). Les quantités d'azote non dégradable du foin et de la paille sont identiques $(1,2 \mathrm{~g} / \mathrm{kg} \mathrm{MS})$, mais ramenée en $\%$ de l'azote total du fourrage, cette fraction est significativement plus élevée pour la paille $(20 \%)$ que pour le foin $(7 \%)$. Cette différence est compensée par une vitesse de dégradation plus élevée de l'azote de la paille. Entre 0 et $1 \mathrm{~h}$ d'incubation, $10 \%$ de l'azote de la paille est dégradé dans le rumen contre seulement $6 \%$ pour le foin. Aussi, le pourcentage d'azote dégradé dans le rumen est-il tout à fait comparable pour les 2 fourrages, soit $61 \%$ pour la paille et $62 \%$ pour le foin, quand le taux de renouvellement des particules dans le rumen est fixé à 0,06 par $h$.

Après traitement à l'ammoniac, la quantité d'azote immédiatement dégradable augmente considérablement, $+10,7 \mathrm{~g} / \mathrm{kg}$ MS pour la paille et $+13,6 \mathrm{~g} / \mathrm{kg}$ MS pour le foin (Tableau III) et représente $62 \%$ de l'azote total pour les 2 fourrages traités (Fig. 1); parallèlement, la quantité d'azote qui se dégrade lentement reste constante ou même diminue dans le cas du foin. Quant à la fraction d'azote non dégradable, elle reste comprise entre 1,0 et 1,3 $\mathrm{g} / \mathrm{kg}$ MS quel que soit le fourrage considéré, traité ou non. En pourcentage de l'azote total, cette fraction ne représente plus que $8 \%$ pour la paille et $4 \%$ pour le foin, contre respectivement 20 et $7 \%$ avant traitement. La vitesse de dégradation des 2 fourrages traités est significativement plus faible que celle des fourrages non traités; entre 0 et $1 \mathrm{~h}$ d'incubation, $2 \%$ de l'azote est dégradé dans le rumen contre respectivement 10 et $6 \%$ pour la paille et le foin non traité. Malgré cette diminution de la vitesse de dégradation, le pourcentage d'azote dégradé dans le rumen passe de 62 avant traitement à $78 \%$ après traitement, cette augmentation étant dûe essentiellement à l'augmentation de la fraction immédiatement dégradable.

\section{DISCUSSION ET CONCLUSION}

La teneur en azote de la paille a augmenté après le traitement à l'ammoniac, de $11 \mathrm{~g} / \mathrm{kg} \mathrm{MS}$, soit une valeur un peu plus élevée que celle rapportée par Chenost et Dulphy (1987) dans leur synthèse bibliographique ( $8 \mathrm{~g} / \mathrm{kg} \mathrm{MS})$, et la teneur en azote du foin est passée de $17,8 \mathrm{~g}$ à 27,7 $\mathrm{g} / \mathrm{kg}$ de MS, ce qui correspond à une fixation de $12 \mathrm{~g}$ d'ammoniac/ $\mathrm{kg}$ de MS, contre

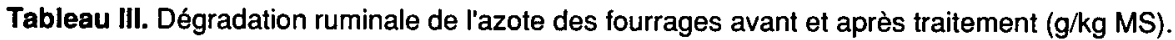

\begin{tabular}{lcccc}
\hline Azote & $\begin{array}{c}\text { Immédiatement } \\
\text { dégradable }\end{array}$ & $\begin{array}{c}\text { Lentement } \\
\text { dégradable }\end{array}$ & $\begin{array}{c}\text { Non } \\
\text { dégradable }\end{array}$ & $\begin{array}{c}\text { Dégradé } \\
\text { dans le rumen }\end{array}$ \\
\hline $\begin{array}{l}\text { Paille } \\
\text { non traitée }\end{array}$ & 0.3 & 5.0 & & \\
traité & 11.0 & 5.4 & 1.3 & 4.0 \\
Foin & & & 1.3 & 13.9 \\
non traité & 3.4 & 13.1 & & \\
traité & 17.0 & 9.6 & 1.2 & 11.1 \\
\hline
\end{tabular}




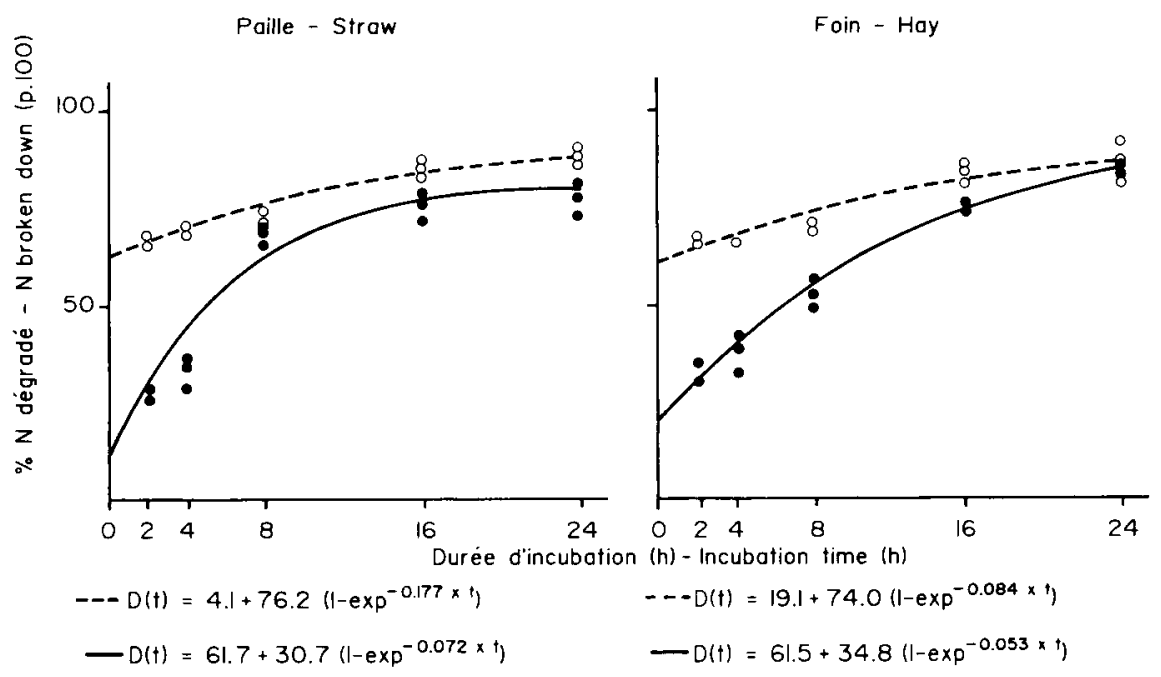

Fig. 1. Cinétique de dégradation de l'azote dans le rumen des fourrages avant $(-\bullet)$ et après traitement $(\mathrm{O}--\mathrm{O})$.

$19 \mathrm{~g}$ trouvé en moyenne par Benahmed et Dulphy (1986) avec des foins humides.

La dégradabilité de l'azote de la paille non traitée est de $61 \%$ dans cet essai, Varvikko et Lindberg (1985) ont trouvé une valeur de dégradabilité de la paille tout à fait comparable (62\%); par contre, Bernard et al. (1988) rapportent une valeur un peu plus élevée $(71 \%)$, la contamination microbienne des résidus de sachets ayant été mesurée dans les 2 essais par marquage de la population bactérienne du rumen avec du ${ }^{15} \mathrm{~N}$. Après traitement à l'ammoniac, la dégradabilité de l'azote de la paille augmente fortement $(78 \%)$, mais cette valeur reste inférieure à celle calculée à partir des résultats de Dryden et Kempton (1983), à savoir $94 \%$. En effet, ces auteurs ne prennent pas en compte dans leur modèle la fraction azotée non dégradable. Par ailleurs, le pourcentage d'azote immédiatement dégradable est un peu plus faible dans notre essai (62\%) que celui rapporté par Dryden et Kempton (74\%).
Cette augmentation de la dégradabilité de l'azote des fourrages traités est due essentiellement à une augmentation de l'azote rapidement solubilisée dans le tampon ou dans le rumen; $75 \%$ de l'azote fixé au cours du traitement se retrouve sous forme d'azote soluble dans notre essai, soit une valeur très proche de celles rapportées par Gordon et Chesson (1983) et Dryden et Kempton (1983), respectivement 73 et $67 \%$. Cette fraction solubilisée correspondrait à la fraction rapidement dégradée qui représente $96 \%$ de l'azote fixé au cours du traitement.

La quantité d'azote indigestible dans le tube digesif (Goering et al., 1972), ou non dégradable par les micro-organismes du rumen (Krisnamoorthy et al., 1983) ou par une protéase simulant l'activité protéolytique ruminale (Pichard, 1977) est liée à la quantité d'azote insoluble dans un solvant acide (N-ADF). Dans notre essai, la quantité d'azote non dégradable n'est pas modifiée par le traitement à l'ammoniac, alors 
que l'azote lié à l'ADF augmente de $0,8 \mathrm{~g} /$ $\mathrm{kg}$ MS pour la paille et de $0,6 \mathrm{~g} / \mathrm{kg} M S$ pour le foin. Cette augmentation de l'azote lié à l'ADF est du même ordre de grandeur que celle rapportée par Dias Da Silva (1986) et Ramihone (1987), sur des pailles traitées à l'urée ou à l'ammoniac. II semblerait donc que, pour les 2 fourrages traités à l'ammoniac, les modifications de répartition de l'azote dans le végétal ne se traduisent pas par des différences de digestion ruminale.

Des 3 hypothèses proposées précédemment pour expliquer l'augmentation anormale de l'azote excrété dans les fèces avec des fourrages traités à l'ammoniac, seule la dernière est en accord avec les résultats obtenus dans cet essai. En effet, le traitement à l'ammoniac des fourrages :

- n'a pas modifié la quantité d'azote non dégradable dans le rumen. L'azote apporté lors du traitement ne semble donc pas se fixer sur les parois indigestibles du fourrage; de même, les protéines du fourrage n'ont pas été rendues, même partiellement, indigestibles par le traitement;

- n'a augmenté que de $10 \%$ la quantité d'azote lentement dégradable de la paille et a même rendu immédiatement dégradable une partie de cette fraction dans le cas du foin. Cette fraction ne représente que 30 à $35 \%$ de l'azote des fourrages traités; aussi, la dégradabilité de l'azote de ces fourrages dans le rumen est-elle peu sensible aux variations d'activité microbienne ou de temps de séjour des particules dans le rumen. D'ailleurs, malgré l'effet favorable d'une complémentation protéique sur la digestibilité de la matière organique des pailles traitées à l'ammoniac (McAllan et Griffith, 1987), les quantités de matières azotées non digérées dans l'ensemble du tube digestif restent élevées avec ce type de fourrage (Ramihone, 1987). Enfin, quand le temps de séjour des particules dans le rumen passe de 24 à $17 \mathrm{~h}$, la dégradabilité in sacco de l'azote des fourrages traités ne diminue que de $4 \%$. L'hypothèse suivant laquelle l'azote fixé serait lié à des parois non digérées dans le rumen ne peut donc être retenue;

- a entraîné une augmentation très importante de la quantité d'azote solubilisé dans une solution tampon, cette fraction représentant à elle seule environ $60 \%$ de l'azote des fourrages traités à l'ammoniac.

Cet azote qui, dans les essais in sacco, disparaît très rapidement, est implicitement considéré comme totalement dégradé dans le rumen. Or seulement 40 à $60 \%$ de cet azote soluble se trouvent sous forme d'ammoniac (Dryden et Kempton, 1983; Gordon et Chesson, 1983; Ramihone, 1987); le reste se trouve sous une forme inconnue. Cette forme d'azote est-elle utilisable par les micro-organismes du rumen? On peut se poser la question, d'autant que des essais réalisés en fermenteur semi-continu semblent montrer qu'une partie de l'ammoniac apporté par le traitement n'est pas utilisé par les microorganismes du rumen (Ramihone, 1987). Cette hypothèse nécessiterait d'être approfondie en étudiant l'utilisation ruminale de l'azote d'une paille traitée à l'ammoniac et préalablement marquée.

\section{REMERCIEMENTS}

Les auteurs remercient $M$. Chenost (INRA) et $G$. Cabon (ITCF) pour les échantillons de paille et de foin qu'ils ont mis à leur disposition. 


\section{RÉFÉRENCES}

Benahmed M. \& Dulphy J.P. (1985) Note sur la valeur azotée des fourrages pauvres traités par l'urée ou l'ammoniac. Ann. Zootech. 34, 335-346

Benahmed M. \& Dulphy J.P. (1986) Influence du traitement des foins à l'ammoniac sur leur valeur azotée appréciée par la méthode des bilans azotés. Ann. Zootech. 35, 387-400

Bernard L., Marvalin O., Yang W. \& Poncet C. (1988) Colonisation bactérienne des différents types d'aliments incubés in sacco dans le rumen: conséquences pour l'estimation de la dégradabilité de l'azote. Reprod. Nutr. Dev. 28, 105-106

Borhami B.E.A. \& Johnson F. (1981) Digestion and duodenal flow of ammonia-treated straw and sodium hydroxide treated straw supplemented with urea, soybean meal or viscera silage. Acta Agric. Scand. 31, 245-250

Chenost M. \& Dulphy J.P. (1987) Amélioration de la valeur alimentaire (composition, digestibilité, ingestibilité) des mauvais foins et des pailles par les différents types de traitement. In : Les Fourrages secs : Récolte, Traitement, Utilisation (Demarquilly C., ed.), INRA, Paris, 199-230

Dias Da Silva A.A. (1986) Urea as a source of ammonia for improving the nutritive value of wheat straw. Anim. Feed Sci. Technol. 14, 6779

Dryden G. McL. \& Kempton T.J. (1983) Digestion of organic matter and nitrogen in ammoniated barley straw. Anim. Feed Sci. Technol. 10, 65-75

Dulphy J.P. \& Demarquilly C. (1981) Problèmes particuliers aux ensilages. In : Prévision de la Valeur nutritive des Aliments des Ruminants. INRA, route de Saint-Cyr, 78000 Versailles, 81 104

Dulphy J.P., Komar A. \& Zwaenepoel P. (1984a) Effets comparés des traitements à l'ammoniac et à la soude sur la valeur alimentaire des fourrages pauvres. Ann. Zootech. 33, 321-342

Dulphy J.P. \& Zwaenepoel P., Komar A. \& Aboulfaraj $S$. (1984b) Valeur alimentaire des foins traités par l'ammoniac. Ann. Zootech. 33 , 187-200

Gordon A.H. \& Chesson A. (1983) The effect of prolongated storage on the digestibility and ni- trogen content of ammonia-treated barley straw. Anim. Feed Sci. Technol. 8, 147-153

Goering H.K., Gordon C.H., Hemken R.W., Waldo D.R., Van Soest P.J. \& Smith L.W. (1972) Analytical estimates of nitrogen digestibility in heat-damaged forages. J. Dairy Sci. 55, 1275-1280

Goering H.K. \& Van Soest P.J. (1970) Forage fiber analysis (apparatus, reagents, procedures and some applications). Agric. Handbk. $\mathrm{N}^{\circ} 379$, Agricultural Research Service, USDA

Krishnamoorthy U., Muscato T., Sniffen C.J. \& Van Soest P.J. (1983) Nitrogen fractions in selected feedstuffs. J. Dairy Sci. 65, 217-225

McAllan A.B. \& Griffith E.S. (1987) The effects of differents sources of nitrogen supplementation of the digestion of fibre components in the rumen of steers. Anim. Feed Sci. Technol. 17, 65-73

Michalet-Doreau B., Vérité R. \& Chapoutot R. (1987) Méthodologie de mesure de la dégradabilité in sacco de l'azote des aliments dans le rumen. Bull. Tech. CRZV Theix, INRA, 69, 5-7

Orskov E.R. \& McDonald J. (1979) The estimation of protein degradability in the rumen from incubation measurements weighted according to rate of passage. J. Agric. Sci. Camb. 92, 499503

Ould-Bah M.Y., Michalet-Doreau B. \& Jamot J. (1988) Colonisation bactérienne des résidus alimentaires des sachets incubés dans le rumen : utilisation du "stomacher" pour la réduire et conséquences sur la mesure de la dégradabilité ruminale de l'azote. Reprod. Nutr. Dev.

Pichard G. (1977) Forage nutritive value. Continuous and batch in vitro rumen fermentations and nitrogen solubility. PhD Thesis, Cornell University, thaca, NY, 91 pp.

Ramihone B. (1987) Facteurs limitant la fermentation dans le rumen des pailles de céréales traitées à l'ammoniac anhydre. Thèse, Univ. des Sciences et Techniques, Languedoc, $85 \mathrm{pp}$.

Solaiman S.G., Horn G.W. \& Owens F.N. (1979) Ammonium hydroxide treatment on wheat straw. J. Anim. Sci. 49, 802-808

Steg A., Den Boer D.J. (1982) Voeder waardschatting van ontsloten rowvoer. Bedrijfsontwikkeling 13, 607-612

Sundstøl T., Coxworth E. \& Mowat D.N. (1978) Improving the nutritive value of straws and other 
low quality roughages by treatment with ammonia. World Anim. Rev. 26, 13-21

Varvikko T., Lindberg J.E. (1985) Estimation of microbial nitrogen in nylon bag residues by food $15 \mathrm{~N}$ dilution. Brit. J. Nutr. 54, 473-481

Vérité R. \& Demarquilly C. (1978) Qualité des matières azotées des aliments pour ruminants.
In: La Vache Laitière. INRA Publ., route de Saint-Cyr, 78000 Versailles, 143-157

Vérité R., Michalet-Doreau B., Chapoutot P., Peyraud J.L. \& Poncet C. (1987) Révision du système des protéines digestibles dans l'intestin (PDI). Bull. Techn. CRZV Theix, INRA 70, 19-34 